\title{
Kinetic Heterogeneities in a Highly Supercooled Liquid
}

\author{
Ryoichi Yamamoto and Akira Onuki \\ Department of Physics, Kyoto University, Kyoto 606-01 \\ (Published in J. Phys. Soc. Jpn. 66, 2545 (1997))
}

\begin{abstract}
We study a highly supercooled two-dimensional fluid mixture via molecular dynamics simulation. We follow bond breakage events among particle pairs, which occur on the scale of the $\alpha$ relaxation time $\tau_{\alpha}$. Large scale heterogeneities analogous to the critical fluctuations in Ising systems are found in the spatial distribution of bonds which are broken in a time interval with a width of order $0.05 \tau_{\alpha}$. The structure factor of the broken bond density is well approximated by the Ornstein-Zernike form. The correlation length is of order $100 \sigma_{1}$ at the lowest temperature studied, $\sigma_{1}$ being the particle size. The weakly bonded regions thus identified evolve in time with strong spatial correlations.
\end{abstract}

Keywords: glass transition, supercooled liquid, heterogeneities, bond breakage

As fluids are supercooled below their melting temperature, their structural relaxations become very slow, resulting in a glass transition at $T=T_{g}$ characterized by a dramatic increase of the viscosity. [1.,2] Recently much attention has been paid to the mode coupling theory, [3, 1, which is a self-consistent decoupling scheme for the density time correlation function and describes onset of glassy slowing down or slow structural relaxations considerably above $T_{g}$. The dominant fluctuation contribution arises from the first peak wave number of the structure factor. For a long time, however, it has been intuitively expected [5 8] that dynamics in glasses should be cooperative, involving many molecules, owing to configuration restrictions. Adam and Gibbs [5] speculated that particle motions over the interparticle distance or the potential barrier can take place only collectively in cooperatively rearranging regions (CRR) and such regions have a minimum size which grows as the temperature is lowered. On the other hand, the mode coupling theory does not predict long range correlations.

In his recent experiments Fischer [9] has reported large excess light scattering with a correlation length $\xi$ $(20-200 \mathrm{~nm})$ which increases on approaching the glass transition from a liquid state. This indicates the presence of large scale heterogeneities in supercooled liquids. To examine this effect, some authors used Monte Carlo simulations on a dense polymer melt. 10,11] There, significant dependence of the diffusion constant on the system size was found, indicating correlations up to the system size. [10] Very recently, Weber et al [11] have found that short range nematic orientational order produces enhancement of long range density correlations. The work of Weber et al suggests that large scale density heterogeneities will not be produced in simple molecular systems.

In their MD simulation on a simple two-dimensional fluid in a deeply supercooled state, Muranaka and Hiwatari 12] visualized significant large scale heterogeneities in particle displacements in a relatively short time interval which corresponds to the so-called $\beta$ relaxation. In liquid states with higher temperatures, Hurley and Harrowell [13 observed similar kinetic heterogeneities but the correlation length was still on the order of a few particle diameters. The characterization of these patterns has not been made in these papers.

It is a natural expectation that structural changes of the configurations of particles occur collectively [5], but it is rather surprising that the visualization of such long range correlations has been nonexistent in the structural or $\alpha$ relaxation. The aims here are to visualize them unambiguously and analyze the patterns qualitatively.

Our system is composed of two different atomic species, 1 and 2, with the numbers $N_{1}=N_{2}=5000$, interacting via the soft-core potential $v_{\alpha \beta}(r)=\epsilon\left[\left(\sigma_{\alpha}+\sigma_{\beta}\right) / 2 r\right]^{12}$, where $r$ is the distance between two particles and $\alpha, \beta=$ 1,2 . We take the size and mass ratios as $\sigma_{2} / \sigma_{1}=1.4$ and $m_{2} / m_{1}=2$. The difference between the particle sizes prevents crystallization. There is no enhancement of the composition fluctuations at any wave number and the system is effectively one-component. The interaction is truncated at $r=4.5 \sigma_{1}$. The leapfrog algorithm [14] is adopted to solve the differential equations with a time step of $0.005 \tau$ under the periodic boundary condition. The space and time are measured in units of $\sigma_{1}$ and $\tau_{0}=\left(m_{1} \sigma_{1}^{2} / \epsilon\right)^{1 / 2}$. The temperature is kept at a constant value using the Gaussian constraint thermostat, 114 and the density is fixed at $n=0.8 / \sigma_{1}^{2}$. We specify the thermodynamic states using the coupling parameter $\Gamma_{\text {eff }}=n\left(\epsilon / k_{\mathrm{B}} T\right)^{1 / 6} \sum_{\alpha, \beta} x_{\alpha} x_{\beta}\left(\sigma_{\alpha}+\sigma_{\beta}\right)^{2} / 4$, 12,15 where $x_{1}$ and $x_{2}$ are the compositions and are equal to $1 / 2$ in our case, so it follows that $\sigma_{\text {eff }} \cong 1.21 \sigma_{1}$ Data are taken at $\Gamma_{\text {eff }}=1.0,1.1,1.2,1.3$ and 1.4. The corresponding temperature is $2.54,1.43,0.850,0.526$ and 0.337 , respectively, in units of $\epsilon / k_{\mathrm{B}}$. The corresponding pressure is $32.8,27.0,23.6,21.63$ and 20.5 , respectively, in units of $\epsilon / \sigma_{1}^{2}$. We realized these states by cooling the system very slowly in a stepwise manner from a liquid state with $\Gamma_{e f f}=0.8$. The equilibration time is 5000 after $\Gamma_{\text {eff }}$ is changed from 1.3 to 1.4. There was no appreciable change in the pressure over computation times of order 10000. The zero-shear viscosity $\eta$ is of order 6 at $\Gamma_{\text {eff }}=1$ and $10^{4}$ at $\Gamma_{\text {eff }}=1.4$ in units of $m_{1} / \tau_{0}$ [16]. It was claimed that glassy behavior becomes apparent for $\Gamma_{e f f} \gtrsim 1.3$. 17]

In our analysis we follow bond breakage processes. For 
each atomic configuration given at time $t_{0}$, a pair of atoms $i$ and $j$ is considered to be bonded if

$$
r_{i j}\left(t_{0}\right)=\left|\mathbf{r}_{i}\left(t_{0}\right)-\mathbf{r}_{j}\left(t_{0}\right)\right| \leq 1.1 \sigma_{\alpha \beta},
$$

where $i$ and $j$ belong to the species $\alpha$ and $\beta$, respectively, and $\sigma_{\alpha \beta}=\frac{1}{2}\left(\sigma_{\alpha}+\sigma_{\beta}\right)$. In our case the particle density is so high that the pair distribution functions $g_{\alpha \beta}(r)$ have a sharp peak at $r \cong \sigma_{\alpha \beta}$. Thus the definition of bonds is very insensitive to the factor in front of $\sigma_{\alpha \beta}$ as long as it is slightly larger than 1 . The polygons, which are composed of the bonds and cover the space, are mostly triangles as shown in Fig. 1. After a lapse of time $t$ we count the number $N_{\text {suv }}(t)$ of surviving bonds which continue to satisfy

$$
r_{i j}\left(t_{0}+t\right) \leq 1.6 \sigma_{\alpha \beta}
$$

If the initially bonded pair does not satisfy (2), we regard it to have been broken. This definition of bond breakage is also insensitive to the factor in front of $\sigma_{\alpha \beta}$ as long as it is considerably larger than 1 and smaller than 2. We have confirmed that $N_{\text {suv }}(t)$ decays exponentially as

$$
N_{\text {suv }}(t)=N_{\text {suv }}(0) \exp \left(-t / \tau_{b}\right) .
$$

This holds excellently in the whole time region for $\Gamma_{\text {eff }} \leq$ 1.2 , but has been obtained from the fit in the short time region $t \lesssim \tau_{b}$ for $\Gamma_{\text {eff }} \geq 1.3$. We have not yet checked whether or not $N_{\text {suv }}(t)$ relaxes exponentially for $t \gtrsim \tau_{b}$. Note that there are three kinds of bonds in our twocomponent fluid, but no significant differences can be found in their breakage processes. In Fig. 2 we show that the bond breakage time $\tau_{b}$ grows strongly with increasing $\Gamma_{\text {eff }}$. Our data may be well fitted to $\tau_{b} \propto T^{-4}$ as well as to $\log \tau_{b} \propto 1 / T$. Because $\tau_{b}$ is the relaxation time of the glassy structures, $\tau_{b}$ should be of the same order as the $\alpha$ relaxation time $\tau_{\alpha}$. In fact for the same model Muranaka and Hiwatari [17] obtained $\tau_{\alpha}$ on the same order as our $\tau_{b}$ from the self part of the time correlation function $F_{s}(q, t)$ at $q=2 \pi / \sigma_{1}$. This coincidence is natural because $F_{s}(q, t)$ at $q=2 \pi / \sigma_{1}$ decays when a tagged particle moves over the interparticle distance.

Fig. 3 displays the bonds broken within the time interval $\left[t_{0}, t_{0}+0.05 \tau_{b}\right]$ at $\Gamma_{\text {eff }}=1.0$ and 1.3 . The center positions $\mathbf{R}_{i j}=\frac{1}{2}\left(\mathbf{r}_{i}\left(t_{0}\right)+\mathbf{r}_{j}\left(t_{0}\right)\right)$ at the initial time $t_{0}$ of the pairs broken within the time interval are depicted here. For the glassy case $\Gamma_{\text {eff }}=1.3$, the broken bond distribution is markedly heterogeneous, being composed of clusters with various sizes, whereas for the liquid case $\Gamma_{\text {eff }}=1$, the inhomogeneity is much weaker. More precisely, in the bond breakage we observe strings of broken bonds involving several particles even in liquid states, because of the high density of our system, and their large-scale aggregation in glassy states. The origin of the heterogeneities is ascribed to the fact that particle motions over the interparticle distance can occur only collectively in glassy states. (5) Furthermore, in Fig. 4 we show the broken bonds in two consecutive time intervals, $\left[t_{0}, t_{0}+0.05 \tau_{b}\right]$ and $\left[t_{0}+0.05 \tau_{b}, t_{0}+0.1 \tau_{b}\right]$, at $\Gamma_{\text {eff }}=1.4$. The initial times at which the bonds are defined are $t_{0}$ and $t_{0}+0.05 \tau_{b}$ for the two cases. We can see that the clusters of broken bonds in the two time intervals mostly overlap or are adjacent to each other. This means that weakly bonded regions or relatively active regions migrate in space on the time scale of $\tau_{\alpha}$. The $\alpha$ relaxation ends when most of the bonds are broken.

We next calculated the structure factor $S_{b}(q)$ of the broken bond density $\rho_{b}(\mathbf{r})=\sum \delta\left(\mathbf{r}-\mathbf{R}_{i j}\right)$ defined by

$$
S_{b}(q)=N_{b}^{-1}\left\langle\left|\sum \exp \left(i \mathbf{q} \cdot \mathbf{R}_{i j}\right)\right|^{2}\right\rangle_{a} .
$$

The summation is over the broken pairs, $N_{b}$ is the total number of the broken bonds, and $\langle\cdots\rangle_{a}$ is the angular average over the direction of the wave vector $\mathbf{q}=$ $(2 \pi / L)\left(n_{x}, n_{y}\right)$ where $n_{x}, n_{y}= \pm 1, \pm 2, \pm 3 . \cdots$. The system length $L$ is 111.8 here. Furthermore, since $S_{b}(q)$ from one configuration fluctuates at small $q$, we have taken the averages of $S_{b}(q)$ from 10 sequential configurations for $\Gamma_{\text {eff }}=1.0-1.3$ and 4 sequential configurations for $\Gamma_{\text {eff }}=1.4$. Then, as shown in Fig.5, $S_{b}(q)$ can be well approximated by the simple Ornstein-Zernike form,

$$
S_{b}(q)=S_{b}(0) /\left[1+\xi^{2} q^{2}\right],
$$

from which we can determine $\xi$. In addition, the raw $S_{b}(q)$ from (4) tends to become 1 at large $q$, so it has been subtracted in $S_{b}(q)$ in Fig. 5. (This subtracted value is negligibly small for $\Gamma_{\text {eff }}>1.1$ at long wavelengths $q \xi<1$, however.) Interestingly,

$$
S_{b}(0) \propto \xi^{2}
$$

and the large $q$ behavior of $S_{b}(q)$ is insensitive to $\Gamma_{\text {eff }}$ as in Ising spin systems near the critical point. In Fig.6 we show $\xi$ vs $1 / T$ for the two widths of the time intervals, $0.05 \tau_{b}(\diamond)$ and $0.1 \tau_{b}(\bullet)$. We can see that $\xi$ is largely independent of the width except for the lowest temperature case, where $\Gamma_{\text {eff }}=1.4$. In this case, however, $\xi$ approaches the system length $L=111.8$ and a finite size effect should be present. Also $\tau_{b}$ at the lowest $T$ in Fig. 2 seems to be affected by the same effect.

Figures 3-5 suggest that the cluster structure in the highly supercooled states comprises clusters with various length scales, the minimum being $\sigma_{1}$ and the maximum being $\xi$. It appears to be self-similar if we consider clusters with size $\ell$ in the region $\sigma_{1} \ll \ell \ll \xi$ in the limit $\xi \gg \sigma_{1}$, which is also supported by the power law behavior $S_{b}(q) \sim 1 / q^{2}$ shown in Fig. 5. This apparently contradicts Adam-Gibbs' phenomenological speculation in which the minimum size of CRR grows upon cooling [5]. We stress that the heterogeneities are of purely kinetic origin and are dynamical objects. Indeed, the small clusters with $\ell \ll \xi$ in Figs. 3 and 4 evolve into larger ones or dissolve in the subsequent time intervals.

We have also calculated the structure factors of the density, composition and stress tensor, but no indication of large scale heterogeneities has been detected in 
such static quantities in accordance with previous papers. 18,19] The kinetic heterogeneities found in this paper play a crucial role in response to externally applied perturbations such as sound waves or shear flow. [16]

A frequently disputed issue in the literature is whether or not there is an underlying thermodynamic phase transition at a nonzero temperature $T_{0}$ below $T_{g}$ in highly supercooled liquids. In our case, Fig. 6 does not indicate any divergence of $\xi$ at a nonzero temperature, although this is not conclusive due to the finite size effect arising from $\xi \sim L$. We mention a recent simulation on a three-dimensional Lennard-Jones mixture by Ghosh and Dasgupta, 20] who used scaling relations of finite systems to conclude $\xi \propto T^{-1.5}$ as $T \rightarrow 0$ without examining cluster structures. The limited data in Fig. 6 also yield $|\partial \log \xi / \partial \log T| \sim 1$.

In summary, by performing long time and large scale MD simulations on a $2 \mathrm{D}$ soft-core mixture, we have examined bond breakage events to find large-scale heterogeneities in the changing rates of the amorphous structure, which are enhanced at low temperatures and surprisingly similar to the critical fluctuations in Ising systems. We believe that this is a first step in the investigation of more complex situations and $3 \mathrm{D}$ cases. We should also clarify the relationship of our patterns in the $\alpha$ relaxation and those by Muranaka and Hiwatari 12 in the $\beta$ relaxation.

We thank Dr. T. Muranaka, Professor Y. Hiwatari and Professor K. Kawasaki for helpful discussions. This work is supported by Grants in Aid for Scientific Research from the Ministry of Education, Science, Sports and Culture. Calculations have been carried out at the Supercomputer Laboratory, Institute for Chemical Research, Kyoto University and the Computer Center of the Institute for Molecular Science, Okazaki, Japan.

[1] J. Jäckle: Rep. Prog. Phys. 49 (1986) 171.

[2] M.D. Ediger, C.A. Angell and S.R. Nagel: J. Phys. Chem. 100 (1996) 13200.

[3] U. Bengtzelius, W. Götze and A. Sjölander: J. Phys. C 17 (1984) 5915.

[4] E. Leutheusser: Phys. Rev. A 29 (1984) 2765.

[5] G. Adam and J.H. Gibbs: J. Chem. Phys. 43 (1965) 139.

[6] M.H. Cohen and G.S. Grest: Phys. Rev. B 20 (1979) 1077.
[7] F.H. Stillinger: J. Chem. Phys. 89 (1988) 6461.

[8] D.N. Perera and P. Harrowell, Phys. Rev. E 54, 1652 (1996).

[9] E.W. Fischer: Physica A 201 (1993) 183.

[10] P. Ray and K. Binder: Europhys. Lett. 27 (1994) 53.

[11] H. Weber, W. Paul and K. Binder: Phys. Rev. Lett. 78 (1997) 2136.

[12] T. Muranaka and Y. Hiwatari: Phys. Rev. E 51 (1995) R2735.

[13] M.M. Hurley and P. Harrowell: Phys. Rev. E 52 (1995) 1694.

[14] M.P. Allen and D.J. Tildesley: Computer Simulation of Liquids, (Clarendon, Oxford, 1987).

[15] B. Bernu, Y. Hiwatari and J.P. Hansen: J. Phys. C 18 (1985) L371.

[16] R. Yamamoto and A. Onuki: preprint

[17] T. Muranaka and Y. Hiwatari: Supplement to Prog. Theor. Phys. 126 (1997) 403.

[18] C. Dasgupta, A.V. Indrani, S. Ramaswamy and M.K. Phani: Europhys. Lett. 15 (1991) 307.

[19] R.M. Ernst, S.R. Nagel and G.S. Grest: Phys. Rev. B 43 (1991) 8070.

[20] S.S. Ghosh and C. Dasgupta: Phys. Rev. Lett. 77 (1996) 1310.

\section{FIGURE CAPTIONS}

Fig.1 Bonds defined at a given time. Their lengths are mostly close to $\sigma_{\alpha \beta}$.

Fig.2 Temperature dependence of the bond breakage time $\tau_{b}(\bullet)$, where $T^{*}=k_{B} T / \epsilon$. The dotted line is a viewing guide. The vertical arrows are estimates for the $\alpha$ relaxation time from the MD data of Muranaka and Hiwatari. 17.

Fig. 3 Snapshots of the broken bonds at $\Gamma_{\text {eff }}=1.0$ and 1.3. The time interval is $0.05 \tau_{b}$, so $5 \%$ of the initial bonds are broken here. The arrows indicate $\xi$.

Fig.4 Broken bond distributions in two consecutive time intervals, $\left[t_{0}, t_{0}+0.05 \tau_{b}\right](\square)$ and $\left[t_{0}+0.05 \tau_{b}, t_{0}+0.1 \tau_{b}\right]$ $(\bullet)$, at $\Gamma_{\text {eff }}=1.4$. The arrows indicate $\xi$.

Fig.5 $S_{b}(q)$ of the broken bond density. The insert shows $1 / S_{b}(q)$ vs $q^{2}$, from which $\xi^{-2}$ is determined.

Fig.6 Temperature dependence of the correlation length $\xi$, where $T^{*}=k_{B} T / \epsilon$. The widths of the time intervals are $0.05 \tau_{b}(\diamond)$ and $0.1 \tau_{b}(\bullet)$. The system length is 111.8 . 
This figure "p66951f01.gif" is available in "gif" format from: http://arxiv.org/ps/cond-mat/9807218v1 
This figure "p66951f02.gif" is available in "gif" format from: http://arxiv.org/ps/cond-mat/9807218v1 
This figure "p66951f03.gif" is available in "gif" format from: http://arxiv.org/ps/cond-mat/9807218v1 
This figure "p66951f04.gif" is available in "gif" format from: http://arxiv.org/ps/cond-mat/9807218v1 
This figure "p66951f05.gif" is available in "gif" format from: http://arxiv.org/ps/cond-mat/9807218v1 
This figure "p66951f06.gif" is available in "gif" format from: http://arxiv.org/ps/cond-mat/9807218v1 